\title{
ORIGINAL
}

\section{PATOLOGÍA TUBERCULOSA ATENDIDA EN URGENCIAS A TRAVÉS DEL ANÁLISIS DEL CMBD DE HOSPITALIZACIÓN EN EL ÁREA DE VALLADOLID OESTE (2002-2006)}

\author{
Raúl López Izquierdo (1), Paula Asensio Villahoz (2), Juan Antonio Vicente Vírseda (3), Isabel \\ González Manzano (1) y M Antonia Udaondo Cascante (4) \\ (1) Servicio de Urgencias del Hospital Universitario Río Hortega. Valladolid. ESPAÑA. \\ (2) Unidad de Codificación Clínica del Hospital Universitario Río Hortega. Valladolid. ESPAÑA. \\ (3) Facultad de Ciencias Económicas y Empresariales. UNED. Madrid. ESPAÑA. \\ (4) Área de la Gerencia de Atención Primaria de Valladolid Este. ESPAÑA
}

\section{RESUMEN}

Fundamento: La Tuberculosis (TB) sigue siendo un problema de salud importante, en muchos casos los servicios de urgencias hospitalarios (SUH) constituyen el primer contacto de los pacientes con el sistema sanitario. Se desconoce el impacto de esta enfermedad en dichos servicios. Los objetivos de este estudio son conocer la importancia de la TB en los SUH y describir las altas hospitalarias (CMBD) con diagnóstico de TB, y cuáles ya habían sido diagnosticadas en el SUH.

Métodos: Estudio descriptivo retrospectivo de las altas con TB en hospitalización y en el SUH de un hospital terciario universitario entre 2002-2006. Estudio de concordancia diagnóstica y análisis de variables según dos grupos: Coincidentes (Alta con TB en el SUH y en el CMBD) y Sólo en el CMBD. Se calculan estadísticos de tendencia central y dispersión para las variables cuantitativas, y proporciones y elaboración de tablas de contingencia con aplicación del test $\mathrm{chi}^{2}$ para las cualitativas.

Resultados: De las $172(0,26 \%)$ urgencias ingresadas que fueron altas hospitalarias con TB, $61(35,5 \%)$ procedían del SUH. La concordancia diagnóstica fue $0,43(43,46 \%)$. Las altas hospitalarias con TB (coincidentes) pasaron de 47 (17) en 2002 a 21 (5) en 2006. El 65,1\% (68,9\% en coincidentes) fueron varones. Por grupos de edad (coincidentes\%): 0-14: 6(33,3\%), 1545: 81(47\%), 46-65: 46(28,7\%), >65: 39(19,7\%); excluyendo de $0-14, \mathrm{p}=0,009$. Localización respiratoria (coincidentes): 110 (50) y baciloscopia positiva (coincidentes): 87 (43). Entre los factores de riesgo conocidos destaca la inmigración ( $\mathrm{p}=0,001)$.

Conclusiones: Las altas con TB descendieron durante el periodo estudiado. La concordancia diagnostica en el SUH fue moderada. Habría que incidir más en personas de mayor edad, y en aquéllas con factores de riesgo conocidos. En el SUH la TB respiratoria es también la más frecuente siendo la baciloscopia el método diagnóstico principal.

Palabras claves: Tuberculosis. Servicio de Urgencias hospitalaria. Factores de riesgo.

Correspondencia:

Raúl López Izquierdo

C/ Fuente Coré 38 Zaratán. 47610 Valladolid

Correo electrónico: rulo636@yahoo.es

\section{ABSTRACT \\ Tuberculosis Pathology Attended in Emergency through the Analysis of the Hospital Discharges MBDS in the West Valladolid Area, Spain (2002-2006)}

Background: Tuberculosis (TB) continues to be an important health problem, in many cases the Hospital Emergency Services (HES) are the first contact of the patients with the sanitary system, there is not known the impact of this disease in the above mentioned services. The objectives of this study are to know the importance of TB in HES and to describe hospital discharges (MBDS) with TB diagnosis and if they had already been diagnosed in the HES.

Methods: A retrospective descriptive study of discharges with TB diagnosis in the hospital ward and in the HES of a tertiary care university hospital from 2002 to 2006. The Diagnostic concordance study and analysis of variables according to two groups: Coincidens (discharge with TB diagnosis in the HES and in the MBDS) and Only in the MBDS. Calculate statisticals of central tendency and dispersal for the quantitative variables and proportions and tables of contingency with application of the test $\mathrm{chi}^{2}$ for the qualitative ones.

Results: Of the $172(0.26 \%)$ emergencies admitted which were hospital discharges with $\mathrm{Tb}$ diagnosis, $61(35.5 \%)$ came in HES. The diagnostic concordance was $0.43(43.66 \%)$. The hospital discharges with $\mathrm{Tb}$ diagnosis (coincidents) changed from 47 (17) in 2002 to $21(5)$ in 2006 . The $65.1 \%(68.9 \%$ in coincidents) were males. As regards age groups (coincidents \%): $0-14: 6(33.3 \%), 15-45: 81(47 \%), 46-65: 46(28.7 \%),>65$ : $39(19.7 \%)$; excluding group $0-14, \mathrm{p}=0.009$. Respiratory location (coincidents): $110(50)$ and positive direct examination of smears for acid-fast bacilli (coincidents): 87 (43). Between well-known risk factors immigration stands out $(\mathrm{p}=0.001)$.

Conclusions: The discharges with TB diagnosis declined during the period studied. The diagnostic concordance in the HES was moderate. We would have to insist more on seniors, and in people with well-known risk factors. In the HES the respiratory TB is also the most frequent being the direct examination of smears for acid-fast bacilli the principal diagnostic method.

Key words: Tuberculosis. Emergency service, hospital. Factors, Risk. 


\section{INTRODUCCIÓN}

La Tuberculosis (TB) constituye un problema de salud importante siendo una enfermedad ampliamente extendida por todo el mundo, y una de las primeras causas de morbimortalidad en los países con un bajo nivel socioeconómico ${ }^{1,2}$.

España, junto a Portugal constituye el país de la Unión Europea con más casos de TB declarados. En los últimos años ha habido un descenso en el número de casos declarados en nuestro país ${ }^{2}$. Los datos así lo demuestran, se ha pasado de declarar 7.753 casos de tuberculosis respiratoria en el año 2000 frente a los 5.851 declarados en el $2006^{3,4}$. A nivel nacional la tasa de notificaciones por 100.000 habitantes de tuberculosis respiratoria también ha descendido pasando de 19,64 casos por 100.000 habitantes en el año 2.000 a 14,74 casos en el año 2006. En la Comunidad Autónoma de Castilla y León el número de casos de tuberculosis respiratorias notificadas ha descendido a casi la mitad, se ha pasando de declarar 520 casos de tuberculosis respiratoria hace 7 años $^{3}$ frente a los 245 en el año 2006 ${ }^{4}$. Actualmente la incidencia en Castilla y León es algo menor que la media nacional: 10,02 casos por 100.000 habitantes $^{4}$. En la ciudad de Valladolid parece que también ha habido un descenso notable en las notificaciones de esta patología. Según datos de un estudio realizado en el Hospital Universitario Río Hortega de Valladolid, durante los años 1996-2000, la incidencia media anual se situaba en 23 casos por 100.000 habitantes $^{5}$ mientras que los datos del Boletín Epidemiológico de Castilla y León indican que en año 2006 hubo un número total de notificaciones de 46 y una incidencia de 8,86 casos por 100.000 habitantes 6 .

De forma tradicional la TB está asociada a situaciones de pobreza, marginalidad, hacinamiento e inmunodepresión tipo SIDA $^{7}$. Por otro lado, hay que destacar que en los últimos años el aumento de la inmigración procedente de países en vías de desarrollo, donde la TB presenta una mayor incidencia que en nuestro país, hace que esta infección sea un problema de salud muy importante entre esta población ${ }^{2}$. Estudios recientes muestran que la infección tuberculosa es más prevalente entre los inmigrantes que entre los autóctonos $^{8,9}$. Los servicios de urgencias hospitalarios (SUH) suponen en muchos casos el primer contacto con el sistema sanitario de muchas personas en situación de riesgo. Estos servicios deberían ser un lugar clave para poder desarrollar una detección y diagnóstico precoz de esta infección, algo básico para controlar la misma a nivel nacional ${ }^{10}$. Es de suponer, por lo tanto, que los profesionales que trabajan en las urgencias de los hospitales deberían mantener un alto índice de sospecha acerca de la posibilidad de una infección tuberculosa. Pero la realidad, es que se desconoce la magnitud del problema que supone la tuberculosis en los SUH.

El objetivo principal que nos proponemos al realizar este estudio es conocer la importancia de la infección tuberculosa en los SUH. Conocer las altas hospitalarias con diagnóstico de TB y cuántas habían sido ya sospechadas o diagnosticadas en el SUH, e investigar el patrón clínico y epidemiológico de estos pacientes.

\section{MATERIAL Y MÉTODOS}

Se realizó un estudio descriptivo retrospectivo de las altas con diagnostico de tuberculosis o sospecha de tuberculosis de cualquier localización anatómica (pulmonar u otras localizaciones) en el Ârea de hospitalización y en el de Urgencias de un hospital de Área universitario de tercer nivel: el Hospital Universitario Río Hortega de Valladolid (HURH), durante el periodo comprendido entre los años 2002-2006. 
Se dispuso de dos fuentes de información:

1. El registro clínico asistencial del
Servicio de Urgencias, cuya descripción/información diagnóstica es cumplimentada por los propios facultativos de urgencias. De donde se seleccionaron las altas cuya descripción diagnóstica incluyera la palabra "tuberculosis o patología tuberculosa" como diagnóstico de alta del SUH. Excluyéndose las que se referían a contactos, historia de TB o antecedentes de TB.

2. El registro del CMBD al alta hospitalaria, de obligada cumplimentación, y que realiza la Unidad de Codificación Clínica del hospital, en donde la información clínica se refleja según la CIE-9$\mathrm{MC}^{11}$, por lo que se seleccionaron las altas atendiendo a la presencia de las categorías comprendidas entre los valores 010-018, correspondientes éstas a las diferentes localizaciones de TB activa como motivo de asistencia. Igualmente se consideró la subclasificación de tales categorías ( $5^{\circ}$ dígito con valor del $\mathrm{O}$ al 6 ) para la identificación del método de diagnóstico de la TB.

Seleccionadas las altas con diagnóstico de TB activa de ambos registros se realizó la comparación de las mismas y determinación de las altas coincidentes entre ellos, para lo cual se consideró que la fecha de atención/alta del Servicio de Urgencias debía coincidir con la fecha de ingreso del registro del CMBD (hospitalización), además de ser un ingreso procedente del Servicio de Urgencias (ingreso urgente). Se revisaron las no coincidentes, consultándose su correspondiente documentación clínica, incluidos resultados del laboratorio, pues la no coincidencia diagnóstica podía deberse a la ausencia de resultados en el momento del alta hospitalaria. Y se realizó un estudio de concordancia diagnóstica entre los dos registros a través del cálculo del Índice Kappa, utilizando la escala propuesta por Landis y Koch para la valoración de la fuerza de dicha concordancia ${ }^{12}$.

Para el análisis de las diferentes variables se consideraron dos grupos:

1. Coincidentes: Diagnosticados de TB en Urgencias con confirmación en el CMBD.

2. Sólo en el CMBD: No diagnosticados de TB en Urgencias pero sí en el CMBD.

Dentro de cada grupo, atendiendo a las variables recogidas en el CMBD de hospitalización, se realizó el análisis de las variables epidemiológicas descritas como relacionadas con la TB: distribución por sexo, edad, lugar de residencia (medio urbano o rural), inmigración, comorbilidades y otros factores de riesgo relacionados, como fumador, bebedor y abuso o/y adicción a otras substancias.

Los datos obtenidos fueron codificados e introducidos en una base de datos Excel. Utilizándose el programa SPSS (Statistical Package for Social Sciences) versión 16 para su análisis estadístico.

El análisis descriptivo consistió en el cálculo de medidas de tendencia central y de dispersión para las variables cuantitativas, y proporciones para las cualitativas. En el caso de estas últimas, además, se obtuvieron las tablas de contingencia, calculando el estadístico Chi-cuadrado. Considerando significativos los valores de $\mathrm{p}<0,05$. Para describir el tipo de asociación detectada se recurre al análisis de la frecuencia esperada en el caso de independencia y de los residuos corregidos, los cuales bajo la hipótesis de independencia estadística siguen una distribución normal, siendo por tanto estadísticamente significativos aquellos mayores de 2 en valor absoluto. 


\section{RESULTADOS}

De las 65.069 personas ingresadas por urgencias en el HURH durante el periodo de estudio, fueron diagnosticadas de TB al alta hospitalaria $172(0,26 \%)$. De éstas $61(35,5 \%)$ ya habían sido diagnosticadas en el SUH. El 6,39\% (11 altas) de todas las altas con TB correspondían a pacientes con más de un episodio asistencial dentro del periodo de estudio.

El resultado del análisis de concordancia diagnóstica entre los dos registros utilizados para la realización del estudio, el del propio SUH y el CMBD de hospitalización, fue un Índice de Kappa de 0,43 $(43,46 \%)$, por lo que atendiendo a la clasificación de Landis y $\mathrm{Koch}^{12}$ podemos afirmar que la concordancia o el grado de acuerdo obtenido es moderado (Kappa grado de acuerdo: < 0 sin acuerdo; De 00,2 insignificante; De 0,2-0,4 bajo; De 0,4-0,6 moderado; De 0,6-0,8 bueno; De 0,8-1 muy bueno).

En la figura 1 se muestran las altas con $\mathrm{TB}$, tanto las diagnosticadas en el SUH y confirmadas en el CMBD, como aquellas que fueron diagnosticadas sólo durante su ingreso hospitalario a lo largo de los años 2002-2006.

En cuanto a la distribución por sexo, del total de personas diagnosticadas de TB, 112 $(65,1 \%)$ fueron hombres frente a 60 mujeres $(34,9 \%)$. No encontrándose diferencias significativas en cuanto a los dos grupos de estudio establecidos (tabla 1).

La edad media de los pacientes con el diagnostico de TB fue de 46,26 años (DS 21,52 ), siendo la del grupo de pacientes diagnosticados en urgencias de 40,13 años (DS 19,51) y la del grupo de los diagnosticados sólo durante el ingreso de 49,63 años (DS 21,89). Según los grupos de edad estudiados, observamos la siguiente distribución: 0-14 años: 3,5\% (6); 15-45: 47,1\% (81); 46-64: 26,7\% (46); >65 años: 22,7\% (39). En la figura 2 se muestra la distribución por grupos de edad en los dos grupos analizados.

Los resultados derivados del análisis de contingencia realizado para medir la posible interacción entre el sexo y la edad de los pacientes (excluyendo el tramo de 0 a 14 años debido al insuficiente número de casos registrados) y la TB, en el SUH o durante la hospitalización, nos revelan

Figura 1

Distribución de las altas con TB por años

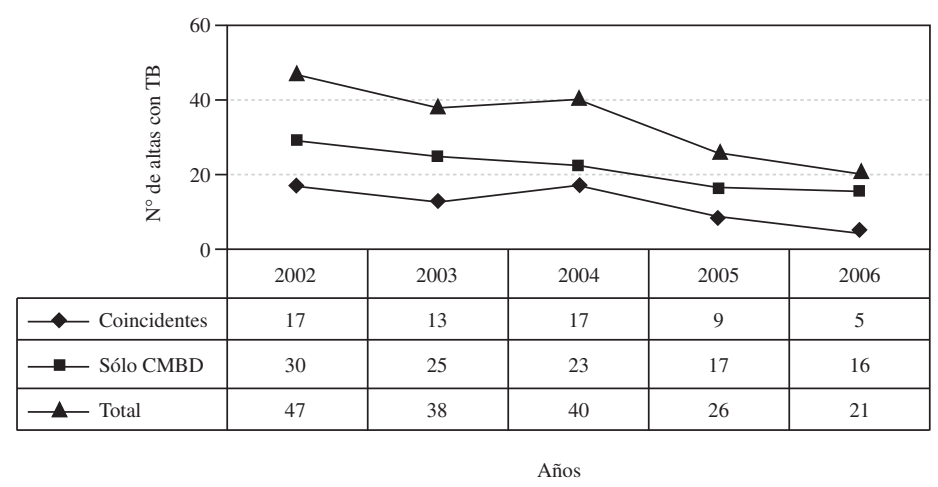


Tabla 1

Factores de riesgo de las altas con $\mathrm{TB}^{*}$

\begin{tabular}{|c|c|c|c|}
\hline & Coincidentes & Sólo CMBD & n \\
\hline & $\mathrm{n}=61(\%)$ & $\mathrm{n}=111(\%)$ & 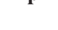 \\
\hline $\begin{array}{l}\text { Sexo } \\
\text { Varón } \\
\text { Mujer }\end{array}$ & $\begin{array}{l}42(68,9) \\
19(31,1)\end{array}$ & $\begin{array}{c}70(63,1) \\
41(36,9)\end{array}$ & 0,446 \\
\hline $\begin{array}{l}\text { Lugar de residencia } \\
\text { Urbano } \\
\text { Rural }\end{array}$ & $\begin{array}{l}40(65,6) \\
21(34,4)\end{array}$ & $\begin{array}{l}72(64,9) \\
39(35,1)\end{array}$ & 0,926 \\
\hline $\begin{array}{l}\text { Lugar de nacimiento } \\
\text { Inmigrante } \\
\text { Autóctono }\end{array}$ & $\begin{array}{l}12(19,7) \\
49(80,3)\end{array}$ & $\begin{array}{c}4(3,6) \\
107(96,4)\end{array}$ & 0,001 \\
\hline $\begin{array}{l}\text { Localización } \\
\text { Respiratoria } \\
\text { Otra localización } \\
\text { Ambas }\end{array}$ & $\begin{array}{c}50(81,9) \\
11(18,1) \\
0\end{array}$ & $\begin{array}{l}60(54) \\
40(36) \\
11(10)\end{array}$ & 0,001 \\
\hline $\begin{array}{l}\text { Fumador } \\
\text { Sí } \\
\text { No }\end{array}$ & $\begin{array}{l}28(45,9) \\
33(54,1)\end{array}$ & $\begin{array}{l}39(35,1) \\
72(64,9)\end{array}$ & 0,383 \\
\hline $\begin{array}{l}\text { EPOC } \\
\text { Sí } \\
\text { No }\end{array}$ & $\begin{array}{c}6(9,8) \\
55(90,2)\end{array}$ & $\begin{array}{l}21(18,9) \\
90(81,1)\end{array}$ & 0,293 \\
\hline $\begin{array}{l}\text { Bebedor } \\
\text { Sí } \\
\text { No }\end{array}$ & $\begin{array}{c}7(11,5) \\
54(88,5)\end{array}$ & $\begin{array}{l}14(12,6) \\
97(87,4)\end{array}$ & 0,977 \\
\hline $\begin{array}{l}\text { Sida/VIH } \\
\text { Sí } \\
\text { No }\end{array}$ & $\begin{array}{c}1(1,6) \\
60(98,4)\end{array}$ & $\begin{array}{c}4(3,6) \\
107(96,4)\end{array}$ & 0,764 \\
\hline $\begin{array}{l}\text { Anemia } \\
\text { Sí } \\
\text { No }\end{array}$ & $\begin{array}{c}3(4,9) \\
58(95,1)\end{array}$ & $\begin{array}{l}23(20,7) \\
88(79,3)\end{array}$ & 0,022 \\
\hline $\begin{array}{l}\text { Abuso y dependencia drogas } \\
\text { Sí } \\
\text { No }\end{array}$ & $\begin{array}{c}5(8,2) \\
56(91,8)\end{array}$ & $\begin{array}{c}12(10,81) \\
97(87,4)\end{array}$ & 0,842 \\
\hline $\begin{array}{l}\text { Desnutrición } \\
\text { Sí } \\
\text { No }\end{array}$ & $\begin{array}{c}3(4,9) \\
58(95,1)\end{array}$ & $\begin{array}{c}11(9,9) \\
100(90,1)\end{array}$ & 0,519 \\
\hline $\begin{array}{l}\text { Diabetes } \\
\text { Sí } \\
\text { No }\end{array}$ & $\begin{array}{c}3(4,9) \\
58(95,1)\end{array}$ & $\begin{array}{c}7(6,3) \\
104(93,7)\end{array}$ & 0,933 \\
\hline $\begin{array}{l}\text { Antecedentes TB } \\
\text { Sí } \\
\text { No }\end{array}$ & $\begin{array}{c}2(3,3) \\
59(96,7)\end{array}$ & $\begin{array}{c}3(2,7) \\
108(97,3)\end{array}$ & 0,977 \\
\hline
\end{tabular}

* Más de un factor de riesgo puede estar presente en un mismo paciente.

ausencia de asociación en el caso del sexo, pero no en la edad, tomando un pvalor para la prueba de la Chi-cuadrado de 0,009 .

En el grupo de los pacientes diagnosticados en el SUH con confirmación en el CMBD, a medida que aumenta la edad de los pacientes la frecuencia observada va tomando valores más bajos con respecto a la esperada (tabla 2), siendo estadísticamente significativos los residuos corregidos en los pacientes de 15 a 45 años y los mayores de 65, obteniéndose por tanto, una mayor concordancia en el diagnóstico de los pacientes de menor edad.

Según el lugar de residencia, medio rural o urbano, la procedencia del medio urbano 
Figura 2

Distribución de las altas con TB por grupos de edad

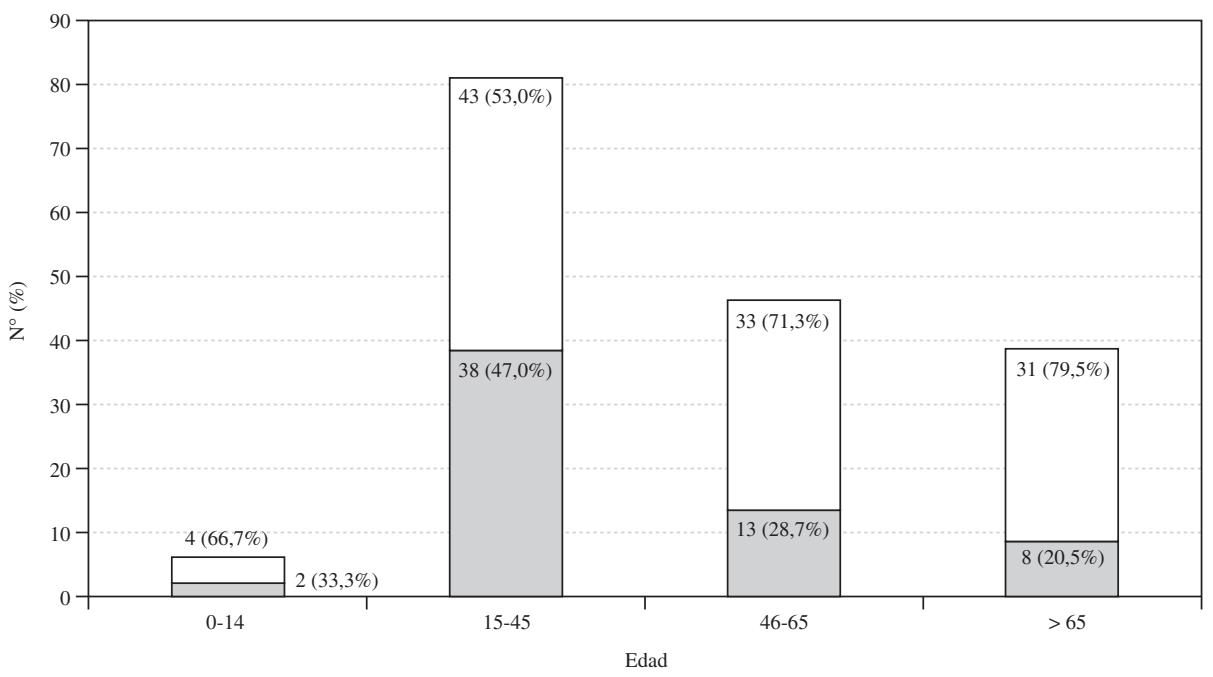

Tabla 2

Tabla de contingencia de los grupos de edad estudiados: estudio de la frecuencia esperada y de los residuos corregidos

\begin{tabular}{|l|c|c|c|c|}
\hline & \multicolumn{3}{|c|}{ Grupos de edad } & \multirow{2}{*}{ Total } \\
\cline { 2 - 4 } & De 15 a 45 & De 46 a 65 & Mayor de 65 & \\
\hline Coincidentes & & & & 5 \\
$\quad$ Recuento & 38 & 13 & 13,9 & 59 \\
Frecuencia esperada & 28,8 & 16,3 & $-2,2$ & 107 \\
Residuos corregidos & 3,0 & $-1,2$ & 31 & 107 \\
\hline Sólo CMBD & 43 & 33 & 25,1 & 166 \\
Recuento & 52,2 & 29,7 & 2,2 & 166 \\
Frecuencia esperada & $-3,0$ & 1,2 & 39 & \\
Residuos corregidos & & & 39,0 & \\
\hline Total & 81 & 46 & & \\
Recuento & 81,0 & 46,0 & & \\
Frecuencia esperada & \multicolumn{2}{|c|}{} \\
\hline
\end{tabular}

fue del $65,1 \%$ (112), con idéntica distribución en ambos grupos (tabla 1).

De todos los pacientes con TB al alta hospitalaria se encontraron que $16(9,3 \%)$ correspondían a inmigrantes, estando el $75 \%$ (12) dentro del grupo de los coincidentes (tabla 1). De las 172 altas con TB 16 tuvieron una afectación múltiple: 2 en el grupo de las coincidentes y 14 en las sólo existentes en el CMBD de hospitalización. Si tenemos en cuenta los códigos diagnósticos existentes en ellas atendiendo a la CIE9-MC ${ }^{11}$ encontramos que en el grupo de las altas con TB procedentes del SUH la afectación fue fundamental respiratoria, en el 
Tabla 3

Distribución de las localizaciones de TB según la CIE-9-MC en el total de las altas hospitalarias*

\begin{tabular}{|l|c|c|}
\hline \multicolumn{1}{|c|}{ Afectación tuberculosa } & Coincidentes & Sólo CMBD \\
\hline 010: Infección tuberculosa primaria & 1 & 0 \\
\hline 011: Tuberculosis pulmonar & 51 & 55 \\
\hline 012: Otras tuberculosis respiratorias & 1 & 16 \\
\hline 013: Tuberculosis de meninges y del sistema nervioso central & 2 & 5 \\
\hline 014: Tuberculosis de intestinos, peritoneo y glándulas mesentéricas & 1 & 8 \\
\hline 015: Tuberculosis ósea y articular & 1 & 7 \\
\hline 016: Tuberculosis del aparato génito-urinario & 0 & 16 \\
\hline 017: Tuberculosis de otros órganos & 1 & 3 \\
\hline 018: Tuberculosis miliar & 5 & 12 \\
\hline Total localizaciones & $\mathbf{6 3}$ & 13 \\
\hline
\end{tabular}

* Desglosadas las afectaciones múltiples.

Tabla 4

Distribución de las localizaciones de TB según el método diagnóstico en el total de las altas hospitalarias

\begin{tabular}{|l|c|c|}
\hline \multicolumn{1}{|c|}{ Método diagnóstico de la TB } & Coincidentes & Sólo CMBD \\
\hline 0: Sin especificar & 7 & 29 \\
\hline $\begin{array}{l}\text { 2: Resultado de examen bacteriológico o histológico desconocido (por el } \\
\text { momento) }\end{array}$ & 6 & 12 \\
\hline 3: Bacilos tuberculosos encontrados (en esputos) mediante microscopia & 43 & 44 \\
\hline $\begin{array}{l}\text { 4: Bacilos tuberculosos no encontrados (en esputos) mediante microsco- } \\
\text { pia, pero sí mediante cultivo bacteriano }\end{array}$ & 36 \\
\hline $\begin{array}{l}\text { 5: Bacilos tuberculosos no encontrados mediante examen bacteriológico, } \\
\text { pero la tuberculosis se ha confirmado por medios histológicos }\end{array}$ & \multicolumn{2}{|c|}{24} \\
\hline $\begin{array}{l}\text { 6: Bacilos tuberculosos no encontrados mediante examen bacteriológico } \\
\text { o histológicos, pero la tuberculosis se ha confirmado mediante otros } \\
\text { métodos }\end{array}$ & 187 \\
\hline Total & 37 & 14 \\
\hline
\end{tabular}

(5º́gito según CIE - 9 - MC: 0-6).

$82,5 \%$ (52) frente al 56,8\% (71) de aquellas procedentes del área de hospitalización (tabla 3).

En el 6,39\% (11) de las altas hospitalarias con TB la afectación tuberculosa múltiple era con afectación respiratoria y otra localización (tabla 1).

En cuanto a la forma de diagnosticar la TB, según lo reflejado en la documentación clínica correspondiente, y atendiendo al total de las localizaciones reflejadas según la $\mathrm{CIE}-9-\mathrm{MC}^{11}$, en el $19,15 \%$ (36) de los casos no estaba especificado. Siendo el estudio micros- cópico (baciloscopia) el método más frecuente entre los pacientes diagnosticados tanto en el SUH 68,25\% (43) como en hospitalización 35,20\%(44) (tabla 4).

Para el análisis de la comorbilidad se realizó el estudio de los diagnósticos secundarios reflejados en el CMBD de hospitalización, obteniéndose una media de diagnósticos secundarios por alta de 5,02, siendo de 3,29 en el grupo de los coincidentes y de 5,97 en el caso de los existentes sólo en el CMBD. Su distribución según los capítulos de la CIE-9-MC ${ }^{11}$ se refleja en la figura 3. 
Figura 3

Diagnósticos secundarios según capítulos de la CIE-9-MC*

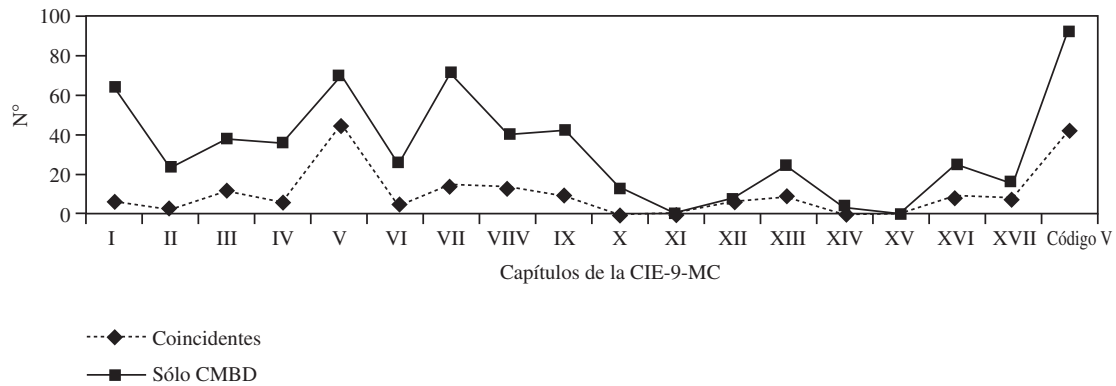

* Capítulos de la CIE-9-MC

I: Enfermedades infecciosas y parasitarias

II: Neoplasias

III: Enfermedades endocrinas de la nutrición, metabólicas y trastornos de la inmunidad

IV: Enfermedades de la sangre y de los órganos hematopoyéticos

V: Trastornos mentales

VI: Enfermedades del sistema nervioso y de los órganos de los sentidos

VII: Enfermedades del sistema circulatorio

VIII: Enfermedades del aparato respiratorio

IX: Enfermedades del aparato digestivo

$\mathbf{X}$ : Enfermedades del aparato genitourinario

XI: Complicaciones del embarazo, parto y puerperio

XII: Enfermedades de la piel y del tejido subcutáneo

XIII: Enfermedades del sistema osteo-mio-articular y del tejido conectivo

XIV: Anomalías congénitas

$\mathbf{X V}$ : Ciertas enfermedades con origen en el periodo perinatal

XVI: Síntomas, signos y estados mal definidos

XVII: Lesiones y envenenamientos

Códigos V: Clasificación suplementaria de factores que influyen en el estado de salud y contacto con los servicios sanitarios

En la tabla 1 podemos observar los resultados obtenidos en relación con los factores de riesgo o entidades clínicas que se asocian más con el diagnóstico de TB dependiendo de si el diagnóstico se ha realizado en el SUH o bien en el estudio hospitalario.

\section{DISCUSIÓN}

El descenso en el número de casos diagnosticados de TB en el periodo estudiado es evidente. En 5 años, el diagnóstico de TB ha descendido más del 50\%, pasando de 47 altas con TB en el año 2002 a 21 en el año 2006. Este descenso se puede comparar con el que se está produciendo en el resto de España como lo indican los últimos estudios epidemiológicos llevados a cabo en nuestro país ${ }^{2,5,13-17}$. Sin embargo, el diagnóstico de TB sigue siendo de gran relevancia en los servicios hospitalarios de nuestro país y aunque a nivel global el porcentaje de estos casos es pequeño éste no se debe considerar despreciable.

La concordancia diagnóstica encontrada en este estudio se puede considerar moderada, esto implica que en los SUH se debería pensar más en esta patología. Es muy importante que se sospeche la infección lo antes posible para no demorar las pruebas diagnósticas e iniciar lo antes posible el tratamiento y también eliminar cuanto antes la posible transmisión de la bacteria por personas bacilíferas.

La gran mayoría de los diagnósticos de TB se realizan en el grupo de edad de 15-45 
años $2,13-16$. En los SUH los diagnósticos mayoritarios también se realizan entre este grupo poblacional.

Un gran número de pacientes diagnosticados se encuentran en el grupo de edad de mayores de 65 años. Además de que la incidencia de infección es mayor en el grupo de mayores de 75 años ${ }^{2,5,13-17}$. Esto contrasta con el pensamiento tradicional de que la TB es una enfermedad de personas jóvenes. En el caso de los pacientes ancianos, nuestros resultados son similares a los de otros estudios españoles, en los que alrededor del $20 \%$ de los diagnósticos de TB se realizan en personas ancianas ${ }^{2,18}$. La Organización Mundial de la Salud (OMS) considera a los ancianos, y más especialmente a los ancianos institucionalizados, como una población de riesgo para el desarrollo y transmisión de esta enfermedad $^{19}$. Las personas de mayor edad tienen un riesgo importante ya que han vivido en épocas donde la enfermedad tenía una importancia muy grande así como una mayor exposición bacilífera. La edad supone también una disminución de la carga inmune por lo que hay una mayor probabilidad de desarrollar la enferme$\mathrm{dad}^{20}$. Las personas de mayor edad que acuden a urgencias lo hacen por signos y síntomas agudos lo que implica que el diagnóstico inicial en el SUH se centre en una patología aguda. Habría que incidir en la búsqueda de TB en las personas de grupos de edad avanzada y poder hacer diagnósticos de una enfermedad que puede estar transmitiéndose de forma silente a través de personas aparentemente no infectadas.

En relación a su distribución por sexo, la gran mayoría de diagnósticos de TB se siguen realizando entre los varones, no observando cambios en la epidemiología a lo largo de los años $2,13,14,17$.

De forma global la TB respiratoria es la más común, al igual que sucede en otras partes del país $2,13,14,17$. Hay que incidir que la TB respiratoria es la forma más diagnosticada en los $\mathrm{SUH}$, siendo los diagnósticos de TB en otras localizaciones mucho menos prevalentes y realizados en estudios más complejos entre los pacientes hospitalizados. El diagnóstico de TB en los SUH se realiza fundamentalmente mediante la baciloscopia del esputo, así la gran mayoría de los pacientes diagnosticados desde urgencias lo fueron mediante esta técnica. La baciloscopia tiene una gran importancia en los SUH. Obtener una baciloscopia positiva en el SUH hace que no demoremos las medidas preventivas, el inicio del tratamiento y el estudio de contactos, tan importante para el control de esta infección ${ }^{10}$.

El factor de riesgo que más se asoció a las altas por TB fue el de fumador, ya hay estudios que evidencian que este hábito está asociado con el riesgo de infectarse por el Mycobacterium tuberculosis y desarrollar TB más severas ${ }^{21}$. Por otra parte, otras entidades como la Diabetes Mellitus $(\mathrm{DM})^{22}$, la adicción a drogas ${ }^{23}$, el alcoholismo $^{24}$, la anemia ${ }^{25}$ o la desnutrición $^{26}$ que condicionan una inmunodepresión ${ }^{7}$, son las que más se asocian con el diagnóstico de TB al alta hospitalaria. Hay que destacar el pequeñísimo número de pacientes diagnosticados de TB que además padecían SIDA y/o VIH, durante el periodo de estudio, aunque el porcentaje es parecido a lo encontrado en zonas similares a la nuestra ${ }^{2,15}$. Este hallazgo indica la baja prevalencia de VIH/SIDA en aquellos pacientes diagnosticados de TB. En la actualidad la introducción y el acceso de los tratamientos antirretrovirales desde hace años ha conseguido un descenso de la incidencia de la TB en estos pacientes ${ }^{23,27}$. Sin embargo, nuestro estudio no engloba el total de los pacientes diagnosticados en el Área, sólo los ingresados, lo cual limita estas conclusiones, ya que es posible que los pacien- 
tes con VIH sean diagnosticados de TB en otros niveles asistenciales como son las consultas especializadas de patología infecciosa.

El factor de riesgo que en la actualidad está modificando la epidemiología de la TB en España es la inmigración ${ }^{28}$. En un estudio del registro regional de casos de TB en la Comunidad Autónoma de Castilla y León, Marcos H. halló que el 4,9\% de las TB diagnosticadas fueron en inmigrantes 6 , algo que contrasta con nuestros resultados que se aproximan al $10 \%$. Estos resultados indican que Valladolid se puede encontrar en una zona media en cuanto al diagnóstico de TB en inmigrantes, muy por encima de la media regional pero sin alcanzar las cifras obtenidas por otros autores, donde la inmigración es un fenómeno mucho más masivo que en nuestra Área de salud. En el año 2004 los datos publicados de las Comunidades Autónomas de Madrid $^{14}$, Cataluña ${ }^{16}$ y Valencia $^{13}$ indican que el porcentaje de TB diagnosticadas en inmigrantes es del $35,1 \%, 28,8 \%$ y $25,2 \%$ respectivamente. Sin embargo, en un estudio publicado en el año 2007 referente a la ciudad de Barcelona, estas cifras ya han aumentado en 3 años hasta el $46,1 \%{ }^{29}$. El fenómeno de la inmigración económica es imparable por lo que es previsible que suba el número de infectados por TB entre los inmigrantes. Los estudios epidemiológicos indican que más de la mitad de aquellos que desarrollan la enfermedad lo harán durante los dos primeros años de su estancia en el país receptor ${ }^{30,31}$, esto implica que desde los SUH se debe estar en alerta e intentar realizar una búsqueda activa de casos para favorecer su diagnóstico precoz. Hay que recalcar que la gran mayoría de los inmigrantes infectados fueron diagnosticados ya en el SUH.

A modo de conclusión podemos decir que la TB se diagnóstica de forma moderada en los SUH y que habría que incidir más en su búsqueda entre las personas de mayor edad, así como en aquellas con factores de riesgo conocidos como la inmigración y todos aquellos que produzcan inmunodepresión. La forma más común de realizar el diagnóstico en los SUH sigue siendo la baciloscopia.

\section{BIBLIOGRAFIA}

1. Global tuberculosis control: surveillance, planning, financing: WHO report 2008. Disponible en http://www.who.int/tb/publications/global_report/ 2008/pdf/fullreport.pdf. (Consultado el 29 de Julio del 2008)

2. Castilla J, Urtiaga M, Hueto J, Sola J, Dorronsoro I, Torroba L, et al. Evolución en las características epidemiológicas de la tuberculosis en Navarra (1994-2003). An Sis Sanit Navar. 2005; 28 (2): 237-45.

3. Situación de enfermedades de declaración obligatoria. España. Año 2000. Disponible en http://www.isciii.es/htdocs/centros/epidemiologia/EDO_series_temporales/EDO2000.pdf. Consultado el 29 de Julio del 2008)

4. Situación de enfermedades de declaración obligatoria. España. Año 2006. Disponible en: http://www.isciii.es/htdocs/centros/epidemiologia/EDO_series_temporales/EDO2006.pdf. (Consultado el 29 de Julio del 2008)

5. Tejero S, Asensio P, Vaquero JL. Vigilancia epidemiológica de la tuberculosis pulmonar atendida en el nivel especializado a partir de dos fuentes de información. Valladolid. Rev Esp Salud Publica. 2003. 77 (2): 211-20.

6. Marcos H. Registro regional de casos de tuberculosis en la comunidad de Castilla y León. Año 2006. Evolución 2003-2006. Boletín epidemiológico de Castilla y León. 2007; 23 (8): 53-60.

7. Perich N. Inmigración y tuberculosis. Vulnerabilidad y rigidez del sistema sanitario. IX Taller Internacional sobre Tuberculosis. UITB-2005. Mesa 6. Enf Emerg. 2005; 7 (4): 196-205.

8. Arce A, Iñigo J, Martínez L, Cabello M, Burgoa Arenales. Tuberculosis e inmigración en un área sanitaria de Madrid. Situación epidemiológica y evolución en la década 1994-2003. Med Clin (Barc). 2005; 125: 210-212. 
9. Sanz B, Blasco T. Características sociodemográficas de la población inmigrante diagnosticada de tuberculosis en la Comunidad de Madrid. Gac Sanit. 2007. 21 (2): 142-146.

10. Propuesta del Grupo de trabajo de expertos en tuberculosis y del Grupo de trabajo de Salud Pública para la prevención y control de la tuberculosis, aprobada por la Comisión de Salud Pública del Consejo Interterritorial del Sistema Nacional de Salud. Plan para la prevención y control de la tuberculosis en España. Disponible en www.msc.es/profesionales/saludPublica/prevPromocion/docs/planTuberculosis.pdf (Consultado el 29 de Julio de 2008).

11. Ministerio de Sanidad y Consumo. Clasificación Internacional de Enfermedades 9a Revisión.

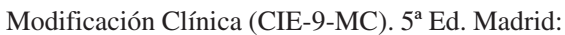
Ministerio de Sanidad y Consumo. 2006.

12. Landis JR, Koch GG. The measurement of observer agreement for cetegorical data. Biometrics. 1977; 33: 159-174.

13. Generalitat Valenciana. Conselleria de Sanitat. Dirección General de Salud Pública. Informes de Salud: $n^{\circ}$ 83. Informe de Tuberculosis. Valencia: Comunidad Valenciana; 2004. Disponible en http://biblioteca.sp.san.gva.es/biblioteca/publicaciones/MATERIAL/PUBLICACIONES/EPI/VIG _EI/TBC/TUBERCULOSIS_04.PDF (Consultado el 11 de Junio de 2008).

14. Registro regional de casos de tuberculosis de la Comunidad de Madrid (informe del año 2004). Disponible en http://www.aamic.es/noticias/documentos/NOTICIA_ENERO_02.pdf (Consultado el 18 de Julio de 2008).

15. Cruz-Ferro E, Fernández E. Epidemiology of tuberculosis in Galicia, Spain, 1996-2005. Int J Tuberc Lung Dis. 11; (10): 1073-79.

16. Alcaide J, Gómez J, López MM, Oyaga N, Plasencia E, Rodés A. Situación epidemiológica y tendencia de la endemia tuberculosa en Cataluña. Informe anual 2004. Catalonia, Spain Direcció General de Salut Pública 2005. Disponible en www.gencat.net/salut/depsan/units/sanitat/pdf/tu ber2003es.pdf (Consultado el 21 de Julio de 2008).

17. García-Zamalloa AM, Arrizabalaga J. Tuberculosis en la comarca del Bajo Deba (Guipúzcoa) en el periodo 1995-2006. Enferm Infecc Microbiol Clin. 2008; 26 (4):187-93.

18. García-Fernandez C, Vera I, Jaén F, Sanz MI, Palenque E, De Juanes JR. Tuberculosis en ancia- nos en un hospital general de la Comunidad de Madrid, 1994-2003. An Med Interna.2006; 23 (4): 161-65.

19. Broekmans JF, Migliori GB, Rieder HL, Leese J, Ruutu P, Loddenkemperc R, et al. European framework for effective tuberculosis control and elimination in countries with a low incidence of tuberculosis. Recommendations of the World Health Organization (WHO), International Union Against Tuberculosis and Lung Disease (IUATLD) and KNCV (Royal Netherlands Tuberculosis Association) Working Group. Eur Resp J. 2002; 19: 765-75.

20. Martín V, Aranzazu M, Ramos J, Otero A, Cortizo J, Travieso S. Incidencia de tuberculosis respiratoria en la provincia de León según el sistema de notificación de enfermedades de declaración obligatoria, 1992-1999. Rev Esp Salud Pública. 2002; 76 (3): 239-48.

21. Hasmiller KM. The association between smoking and tuberculosis. Salud Pública Mex. 2006; 48; Suppl 1: 201-16.

22. Jeon CY, Murray MB (2008) Diabetes Mellitus Increases the Risk of Active Tuberculosis: A Systematic Review of 13 Observational Studies. PLoS Med 5 (7): e152 doi:10.1371/journal.pmed.0050152

23. Velasco M, Castilla Y, Cervero M, Sanz J, Condens E, Gaspar G, et al. The changing pattern of tuberculosis and HIV co-infection in inmigrants and Spaiards in the last 20 years. HIV Medicine. 2008; 9: 227-33.

24. Anibarro L, Lires JA, Iglesias F, Vilariño C, Baloria A, de Lis $\mathrm{JM}$ et al. Factores sociales de riesgo para la falta de cumplimiento terapéutico en pacientes con tuberculosis en Pontevedra. Gac Sanit. 2004; 18 (1): 38-44.

25. Sei Won Lee, Young Ae Kang, Young Soon Yoon, Sang-Won Um, Sang Min Lee, Chul-Gyu Yoo et al. The Prevalence and Evolution of Anemia Associated with Tuberculosis. J Korean Med Sci. 2006; 21: 1028-32.

26. Schwenk A, Macallan DC. Tuberculosis, malnutrition and wasting. Curr Opin Clin Nutr Metab Care. 2000 Jul; 3 (4):285-91.

27. Úriz J, Repáraz J, Castiello J, Sola J. Tuberculosis en pacientes infectados por el VIH. An. Sist. Navar. 2007; 30, Supl 2: 131-42.

28. Grupo de Trabajo de los Talleres de 2001 y 2002 de la Unidad de Investigación en Tuberculosis de 
Barcelona. Prevención y Control de las tuberculosis importadas. Med Clin (Barc). 2003: 121 (14): 549-62.

29. Orcau A, Rius C, García P, Caylà J. La tuberculosis a Barcelona. informe 2007 programa de prevenció i control de la tuberculosi de Barcelona. Disponible en http://www.aspb.es/quefem/docs/Tuberculosi_2007.pdf
30. Junyent M, Núñez S, Miró O. Urgencias médicas del inmigrante adulto. An. Sist. Sanit. Navar. 2006; 29, Supl 1: 27-34.

31. Diz S, López-Velez R, Moreno A, Fortún J, Moreno L, Gómez-Mampaso E et al. Epidemiology and clinical features of tuberculosis in immigrants at an infectious diseases department in Madrid. Int J Tuberc Lung Dis. 2007; 11 (7): 769-74. 